\begin{tabular}{|c|l|}
\hline Title & Carrier Dynamics in a Series of Organic Magnetic Superconductors \\
\hline Author(s) & Naito, Toshio; Matsuo, Saori; Inabe, Tamotsu; Toda, Y asunori \\
\hline Citation & $\begin{array}{l}\text { Journal of Physical Chemistry C, 116(3), 2588-2593 } \\
\text { https:/doi.org/10.1021/jp210966u }\end{array}$ \\
\hline Issue Date & 2012-01-26 \\
\hline Doc URL & http://hdl.handle.net/2115/48386 \\
\hline Type & article \\
\hline File Information & JPCC116-3_2588_2593.pdf \\
\hline
\end{tabular}

Instructions for use 


\title{
Carrier Dynamics in a Series of Organic Magnetic Superconductors
}

\author{
Toshio Naito, $^{*+,}{ }^{\S}$ Saori Matsuo, ${ }^{\dagger}$ Tamotsu Inabe, $^{\dagger}$ and Yasunori Toda ${ }^{\ddagger}$ \\ ${ }^{\dagger}$ Division of Chemistry, Graduate School of Science, Hokkaido University, Sapporo, Hokkaido, 060-0810 Japan \\ ${ }^{\ddagger}$ Graduate School of Engineering, Hokkaido University, Sapporo, Hokkaido, 060-8628 Japan \\ Supporting Information
}

\begin{abstract}
Understanding how electrons behave, i.e., carrier dynamics, in superconductors is indispensable for understanding the mechanism of superconductivity. Recently we have reported the carrier dynamics of $\kappa$ - and $\lambda$-(BETS $)_{2} \mathrm{MCl}_{4}(\mathrm{BETS}=$ bis(ethylenedithio)tetraselenafulvalene, $\mathrm{M}=\mathrm{Ga}, \mathrm{Fe}$ ) based on ultrafast spectroscopy, but the interpretation of the results remains an open question. In this paper we interpreted the results with the aid of newly measured magnetic susceptibility, X-ray single crystal structural analysis, and band calculation. Observa-
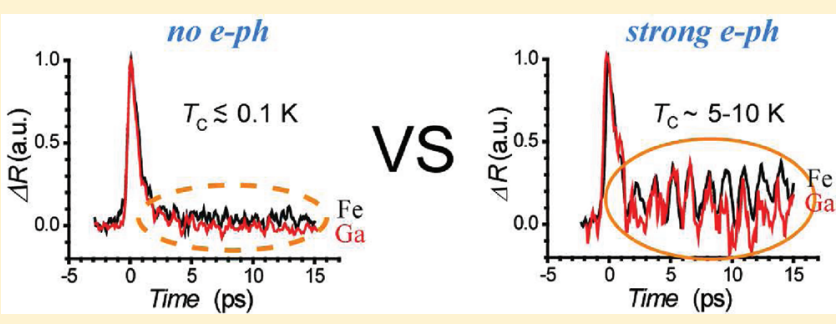

tion of coherent phonons only in the $\lambda$-type salts indicated that $e$-ph interaction should be characteristically strong in the $\lambda$-type salts. By comparison of the observed carrier dynamics with a two-temperature model, the temperature-dependence of carrier dynamics is consistently explained by the different strengths of the $e$-ph interaction between the $\lambda$ - and the $\kappa$-type salts. The difference in strength of the $e$-ph interactions is related to their crystal structures. In conclusion, their carrier dynamics is consistently interpreted and classified by their crystal structures.
\end{abstract}

\section{INTRODUCTION}

Among various kinds of superconductors (SCs) found to date, organic SCs form a major group $(\sim 120$ organic SCs known $)$ and are still increasing both in number and variety. ${ }^{1}$ They enable clear discussion because of well-defined chemical compositions and simple structures, because they become (super)conducting without doping. Although their critical temperatures $\left(T_{C} s\right)$ are low, they have been providing interesting and valuable problems suggesting new or general superconducting mechanisms and how to enhance the $T_{C}$ s. Among the organic SCs (OSCs), the latest and most interesting is a series of charge-transfer (CT) salts of BETS, i.e., bis(ethylenedithio)tetraselenafulvalene (Chart 1). ${ }^{2-4}$ BETS can produce unique and various electronic systems depending on its anions in the $\mathrm{CT}$ salts. In the $\mathrm{CT}$ salts the radical anionic BETS forms conduction pathways by their close interchalcogen-atom, i.e., $S(\mathrm{e})-\mathrm{S}(\mathrm{e})$, network. An interesting point is the fact that one can control their (super)conducting properties by varying the anions, which are not responsible for the electrical conduction. This is sometimes because there is significant interaction between BETS and the anions and sometimes because the crystal lattices vary according to the anions, which in turn varies the molecular arrangement of BETS. Now many scientists are interested in their electronic structures and properties in terms of correlated electronic systems, interaction between conduction electrons and localized spins, and magnetic ordering with (simultaneous or successive) superconducting transition, because some of them are not realized or rare in other group of SCs. ${ }^{4-10}$ Thus, in terms of solid state physics and chemistry, direct observation of their carrier dynamics and a detailed comparative study would provide important information, which one could not obtain otherwise. However, the CT
Chart 1. Molecular Structures of BETS and ET

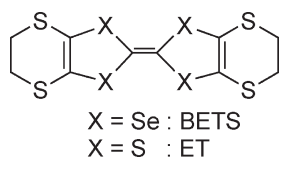

salts of BETS are difficult to obtain because of its difficulty of synthesis, in contrast to the well-known and commercially available donor molecule ET (bis(ethylenedithio)tetrathiafulvalene, Chart 1), which forms the majority of OSCs. ${ }^{1}$ Therefore, as for the BETS salts, the electrical behavior under high pressure and/or magnetic field is intensively studied by worldwide collaboration, ${ }^{8-10}$ while many of other physical properties remain to be studied for $\sim 20$ years. The next important step includes explaining their electrical properties by measuring other physical properties and interpreting all of them in a consistent way in order to design the OSCs with higher $T_{\mathrm{C}}$.

Among various kinds of experimental methods to probe how carriers behave (carrier dynamics) in the organic superconductors, time-resolved measurements are powerful and unique methods in that they enable us direct observation of the behavior. ${ }^{11-15}$ We recently reported on a time-resolved optical study on a series of the BETS magnetic and superconducting salts: ${ }^{16,17} \kappa$-(BETS $)_{2}$ $\mathrm{FeCl}_{4}\left(T_{\mathrm{C}}=0.1 \mathrm{~K} \text {, Néel temperature } T_{\mathrm{N}}=0.5 \mathrm{~K}\right)^{18}$ and $\lambda$-(BETS $)_{2} \mathrm{FeCl}_{4}\left(T_{\mathrm{C}}=1.8 \mathrm{~K}(3.0 \mathrm{kbar}), T_{\mathrm{N}}=8.5 \mathrm{~K}\right)^{19}$

Received: November 14, 2011

Revised: December 8, 2011

Published: December 20, 2011 
(a)

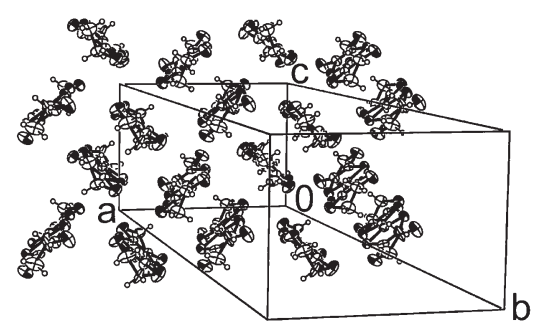

(b)

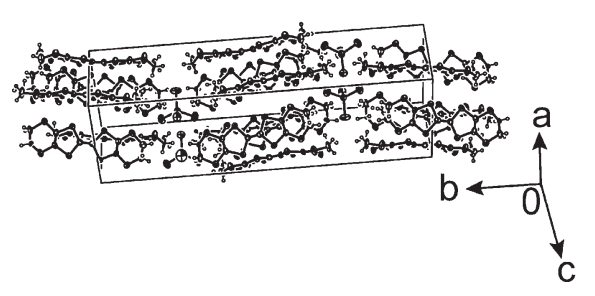

(c)

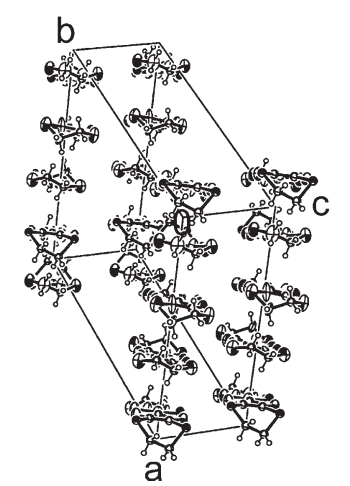

(d)

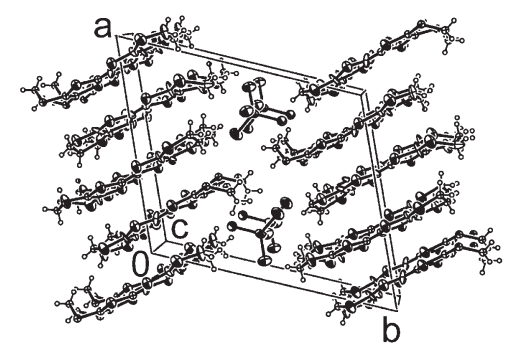

Figure 1. Crystal structures of (a and b) $\kappa$ - and (c and d) $\lambda$-(BETS $)_{2}$ $\mathrm{MCl}_{4}(\mathrm{M}=\mathrm{Ga}, \mathrm{Fe})$. In (a) and (c), the $\mathrm{MCl}_{4}{ }^{-}$anions are omitted for clarity to show the donor molecular arrangements in the conduction sheets.

(respectively abbreviated as $\kappa$ - and $\lambda-\mathrm{FeCl}_{4}$ below) and the nonmagnetic counterparts $\kappa$-(BETS $)_{2} \mathrm{GaCl}_{4}(\text { metallic }>0.5 \mathrm{~K})^{19}$ and $\lambda$-(BETS $)_{2} \mathrm{GaCl}_{4}\left(T_{\mathrm{C}}=4-8 \mathrm{~K}\right)^{19}\left(\kappa-, \lambda-\mathrm{GaCl}_{4}\right)$. All of the $\kappa$ - and the $\lambda$-type salts, respectively, have almost identical crystal structures to one another irrespective of the anions (Figure 1). The only difference lies in the local spins on the $\mathrm{MCl}_{4}$ monoanions; $\mathrm{GaCl}_{4}(S=0)$ is nonmagnetic, while $\mathrm{FeCl}_{4}(S=1 / 2)$ is magnetic. The $\kappa$-type crystal structure/donor molecular arrangement is practically identical to that of ET-based SCs having the highest $T_{\mathrm{C}} \mathrm{s}(\sim 10 \mathrm{~K})$ in OSCs. However, only a few of the $\kappa$-type BETS salts become $\mathrm{SCs}$ with significantly lower $T_{\mathrm{C}} \mathrm{S}(\sim 0.1 \mathrm{~K})$ than those of $\kappa$-ET SCs; the rest of them remained metallic down to
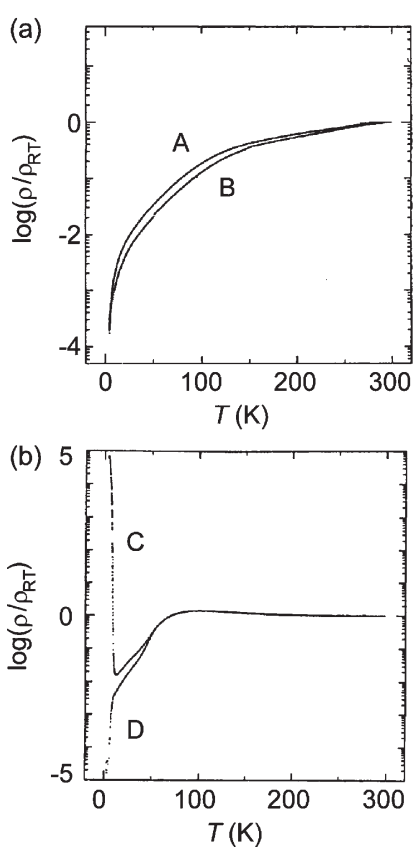

Figure 2. Temperature dependences of resistivity of (a) $\kappa$ - and (b) $\lambda$-(BETS $)_{2} \mathrm{MCl}_{4}(\mathrm{M}=\mathrm{Ga}, \mathrm{Fe})$.

the lowest temperature of measurements $(<1 \mathrm{~K})$ (Figure 2). Meanwhile, most researchers consider that the interaction $(\pi-\mathrm{d}$ interaction) between conduction electrons ( $\pi$ electrons) from the BETS molecules and localized electrons on the Fe atoms (d electrons) should play an important role in their occurrence/ disappearance of superconductivity in $\kappa$ - and $\lambda$-(BETS $)_{2} \mathrm{FeCl}_{4}$ salts. In this way, they have many aspects to be considered for superconducting mechanism; their phase diagrams are complicated, and classification of the electrical behavior is never straightforward in terms of the structures and the localized spins. This is why they have been a center of interest since the first report ${ }^{3,4,19}$ and have been the (challenging) sample of choice in studying the relation between the crystal/electronic structures and superconductivity and in studying the role of localized spins in the mechanism of superconductivity. In our previous work, using a two-color pump - probe method with a time resolution of $\sim 150 \mathrm{fs}$, we directly observed and compared their carrier dynamics at $\sim 7-100 \mathrm{~K}$. On the basis of the obtained temperature dependence of relaxation time $\tau(T)$, amplitude of optical response $A(T)$, and offset $C(T)$, we concluded that the carrier dynamics was classified by the crystal structures in $\kappa$ - and $\lambda$-(BETS $)_{2} \mathrm{MCl}_{4}(\mathrm{M}=\mathrm{Ga}, \mathrm{Fe}) .{ }^{16}$ Accordingly, the following questions remained open: (A) why carrier dynamics differs depending on the crystal structures and (B) why the $\lambda$-type BETS salts generally exhibit much higher transition temperatures $\left(T_{C}, T_{N}\right)$ than those of the $\kappa$-type BETS salts. In this paper, we will answer these questions on the basis of carrier dynamics with the help of the magnetic susceptibility and tight-binding band structure calculation of $\kappa$ - and $\lambda$-(BETS $)_{2} \mathrm{MCl}_{4}(\mathrm{M}=\mathrm{Ga}, \mathrm{Fe})$.

\section{EXPERIMENTAL METHODS}

The BETS molecule and the single crystals of the charge transfer salts were prepared according to the literature. ${ }^{2-4,20}$ Tightbinding band structures were calculated using Caesar 2.0. ${ }^{21}$ All the atoms in the BETS molecules (carbon, hydrogen, sulfur, and selenium atoms) are considered in the calculation, while the 


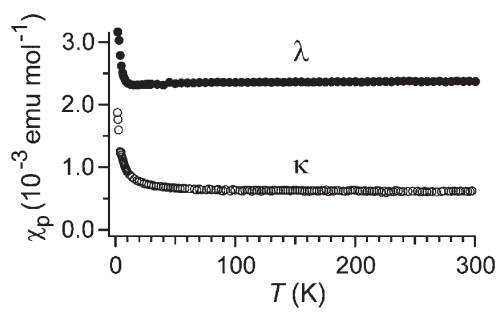

Figure 3. Temperature dependences of magnetic susceptibility of $\kappa$ - and $\lambda$-(BETS) ${ }_{2} \mathrm{GaCl}_{4}$ (measured under $H=1[\mathrm{~T}]$ ).

atoms in the $\mathrm{MCl}_{4}{ }^{-}$ions $(\mathrm{M}=\mathrm{Ga}, \mathrm{Fe})$ are ignored. Validity of ignoring $\mathrm{MCl}_{4}^{-}$anions is supported by the observed magnetic behavior, which is discussed below. Because the resultant band structures were generally dependent on parameters, four different parameter sets of Slater type orbitals were examined in this work (Tables S1-S5 in Supporting Information). Atomic parameters necessary for the band calculation were obtained from single crystal X-ray structural analyses in this work (Tables S6-S15 in Supporting Information). The obtained crystal data agreed with those in previous reports. ${ }^{2-4,20}$ Magnetic susceptibility were measured at 300-2 K with MPMS-XL (Quantum Design). At each run a polycrystalline sample of $1-5 \mathrm{mg}$ was put in a polystyrene straw, which was briefly heated and closed at its middle point in advance and set at the end of the cryostat using another polystyrene straw and then subjected to typical magnetic fields of 500-5000 G during the measurements. Some samples were measured in a gelatin capsule packed with quartz wool, which was set in a middle of the polystyrene straw. The diamagnetic contribution from the straws and BETS $\left(-2.12 \times 10^{-4} \mathrm{emu} \mathrm{mol}{ }^{-1}\right)$ were independently measured $(250-300 \mathrm{~K}$ at every $10 \mathrm{~K}$ and then averaged) and subtracted for correction. Similarly, the diamagnetic contribution from $\mathrm{GaCl}_{4}^{-}\left(-1.07 \times 10^{-4} \mathrm{emu} \mathrm{mol}{ }^{-1}\right)$ were estimated by the similar superconducting quantum interference device (SQUID) measurements of $\left\{\left(\mathrm{C}_{2} \mathrm{H}_{5}\right)_{4} \mathrm{~N}\right\} \mathrm{GaCl}_{4}(-2.14 \times$ $\left.10^{-4} \mathrm{emu} \mathrm{mol}{ }^{-1}\right)$ and $\left\{\left(\mathrm{C}_{2} \mathrm{H}_{5}\right)_{4} \mathrm{~N}\right\} \mathrm{I}\left(-1.59 \times 10^{-4} \mathrm{emu}\right.$ $\left.\mathrm{mol}^{-1}\right)$, using the molar diamagnetic susceptibility of $\mathrm{I}^{-}(-5.2 \times$ $10^{-5}$ emu mol ${ }^{-1}$ ) from a database. ${ }^{22}$ As for the $\mathrm{FeCl}_{4}{ }^{-}$ions, the values in the database ${ }^{22}$ were used for the diamagnetic correction $\left(\mathrm{FeCl}_{4}{ }^{-} ;-1.14 \times 10^{-4} \mathrm{emu} \mathrm{mol}^{-1}\right)$. In the fitting analysis of the $\mathrm{FeCl}_{4}$ salts, the inverse of Curie-Weiss formula

$$
\chi_{\mathrm{p}}^{-1}=\frac{T-\theta}{C}
$$

was applied to the inverse of paramagnetic susceptibility $\chi_{\mathrm{p}}{ }^{-1}$ vs temperature $T$ plot to derive the Curie constant $C$ and Weiss temperature $\theta$ of each salt. As for $\kappa$ - and $\lambda-\mathrm{GaCl}_{4}$, their paramagnetic susceptibility $\chi_{\mathrm{p}}$ was analyzed assuming the following fitting equation for either salt

$$
\begin{aligned}
\chi_{\mathrm{p}} & =\chi(\text { Pauli })+\chi(\text { Curie }) \\
& =\chi(\text { Pauli })+\frac{C}{T}
\end{aligned}
$$

where $\chi$ (Pauli) and $C$ mean Pauli paramagnetism because of the sample and Curie constant because of impurities. On some of the BETS salts in this study, their magnetic properties are partly reported, and our results are consistent with them (Table S16 of Supporting Information). As some sample dependences in the $T_{\mathrm{C}}$ of $\lambda-\mathrm{GaCl}_{4}$ were reported, the average $T_{\mathrm{C}}$ of our sample was
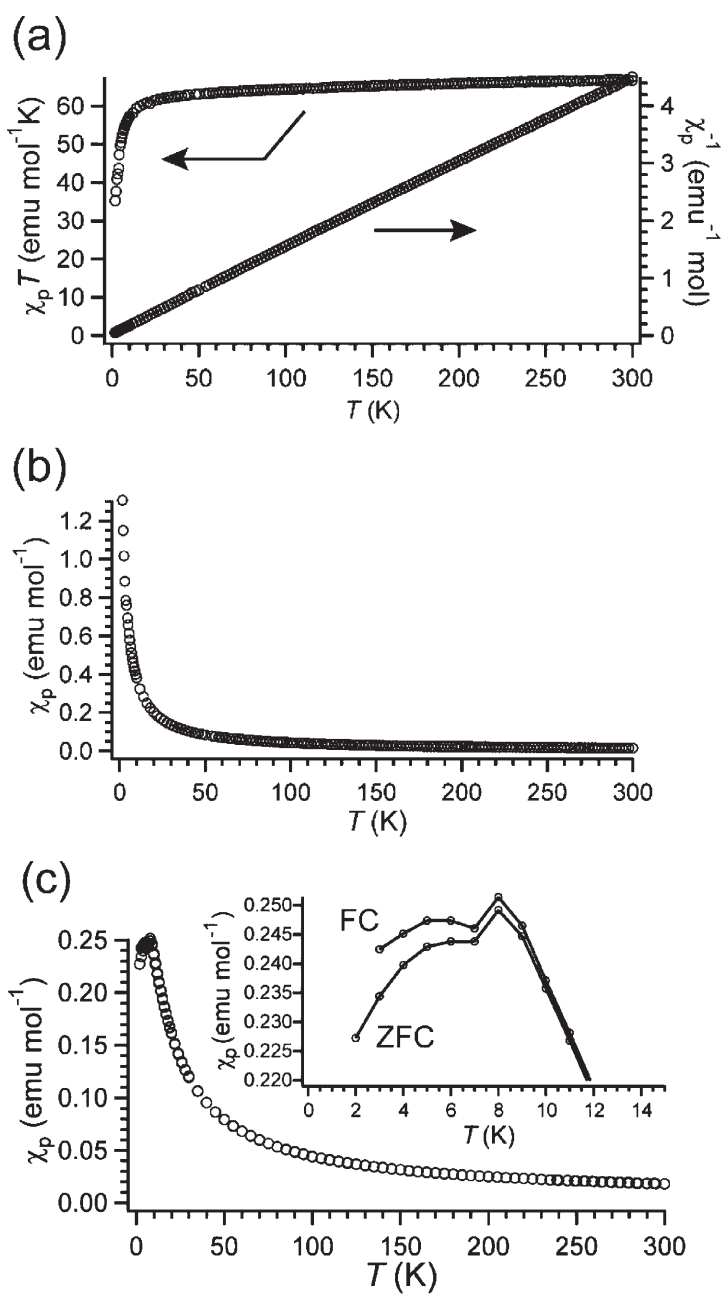

(d)

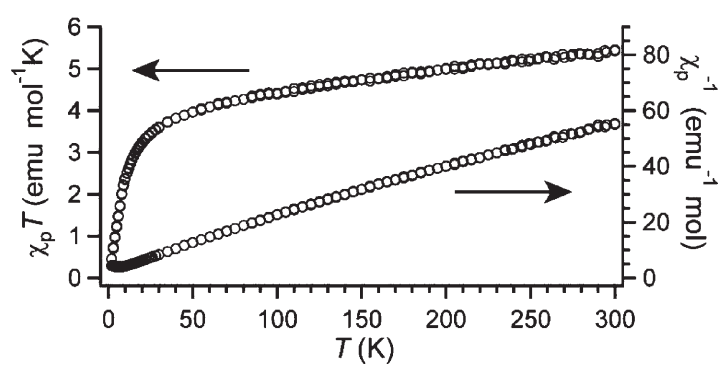

Figure 4. Temperature dependences of magnetic susceptibility of (a and b) $\kappa$ - (measured under $H=1[\mathrm{~T}]$ ) and (c and d) $\lambda$-(BETS) ${ }_{2} \mathrm{FeCl}_{4}$ (measured under $H=500[\mathrm{G}]$ ). The same data are shown in some different types of plots, respectively, in order to emphasize subtle difference in strength of magnetic interactions among materials.

checked by the Meissner effect (Figure S1 of Supporting Information) and confirmed $T_{\mathrm{C}} \approx 4 \mathrm{~K}$ (onset).

\section{RESULTS AND DISCUSSION}

3.1. Magnetic Susceptibility: Estimation of $e-e$ and $e$-ph Interactions. The nonmagnetic counterparts $\left(\kappa-, \lambda-\mathrm{GaCl}_{4}\right)$ exhibited a Pauli paramagnetism $\chi$ (Pauli) of $6 \times 10^{-4}$ to $2 \times$ $10^{-3} \mathrm{emu} \mathrm{mol}^{-1}$ in their metallic phases (Figure 3 ). To estimate the many-body effect ( $e$ - $e$ and/or $e$-ph interactions) as deviation from the tight-binding model, we derived the density of states 


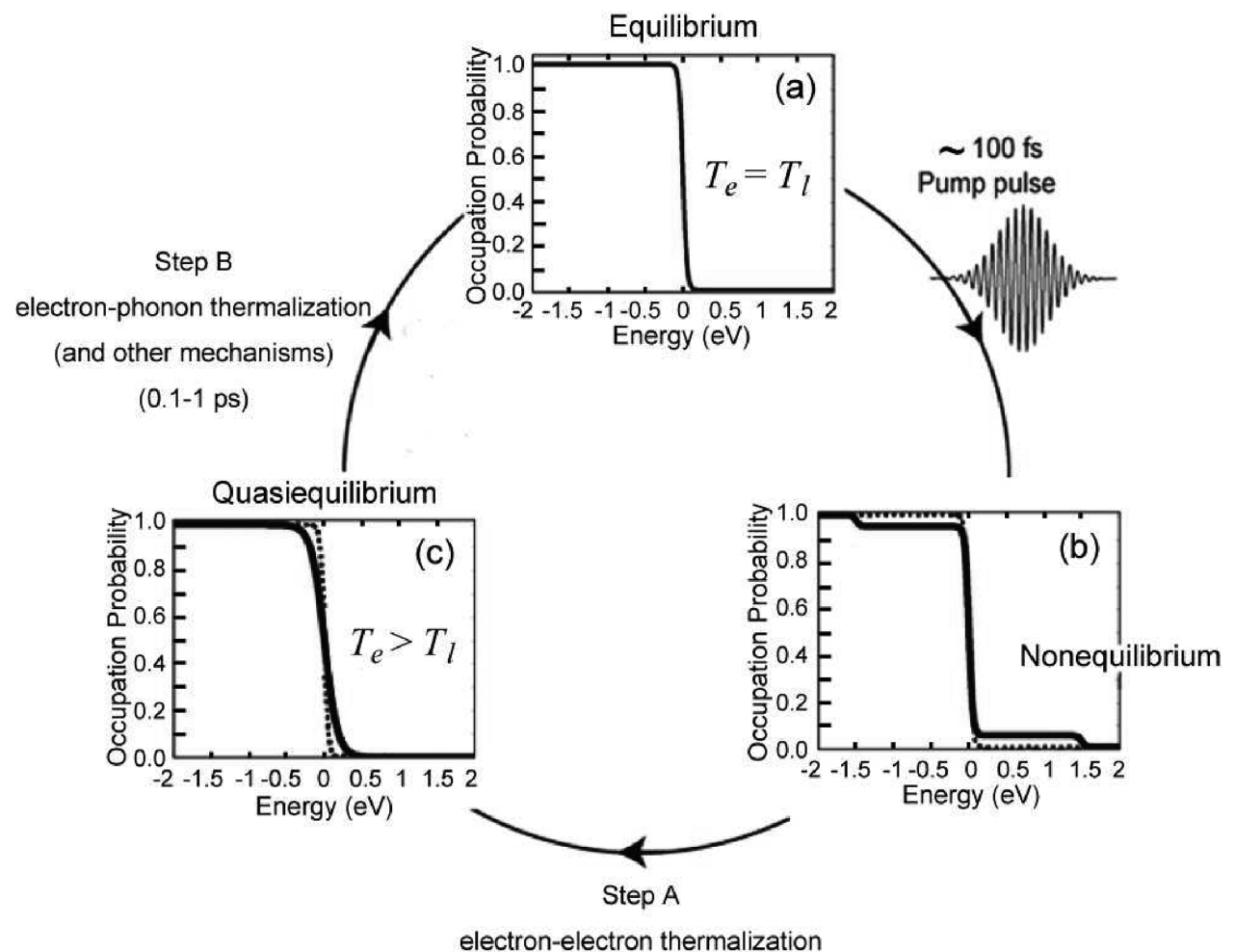

(10's of fs )

Figure 5. Schematic illustration of pump-probe process in the TTM. Produced based on a similar figure in ref 25 .

at the Fermi levels $D\left(E_{\mathrm{F}}\right)$ from these observed values $\chi$ (Pauli) assuming a Fermi liquid model

$$
\chi(\text { Pauli })=g \mu_{\mathrm{B}}^{2} D\left(E_{\mathrm{F}}\right)
$$

which gave $4-5 \times 10^{22} \mathrm{eV}^{-1} \mathrm{~cm}^{-3}$ for both $\kappa$ - and $\lambda-\mathrm{GaCl}_{4}$. These values are several to ten times as large as the calculated values (4-9 $\times 10^{21} \mathrm{eV}^{-1} \mathrm{~cm}^{-3}$, depending on the parameters) based on tight-binding approximation. Both $\kappa$ - and $\lambda$-type salts exhibited typical Pauli paramagnetic behavior but relatively large values of $\chi$ (Pauli); there should be a many-body effect, i.e., electronphonon (e-ph) and/or electron-electron (e-e) interactions in these salts. By comparison of each absolute value of $\chi$ (Pauli), the $\lambda$-type salts exhibit slightly larger values $\left(2.2 \times 10^{-3} \mathrm{emu} \mathrm{mol}^{-1}\right)$ than those of the $\kappa$-type salts $\left(6.1 \times 10^{-4} \mathrm{emu} \mathrm{mol}{ }^{-1}\right)$. As discussed below, this difference might be attributed to the strong $e$-ph interaction characteristic to the $\lambda$-type crystal structure. However, we could not rule out the possibility that a strong e-e interaction should also be important or rather should dominate in the $\lambda$-type salts at this time.

We will make a brief comment on the magnetic behavior of $\kappa$ - and $\lambda$-(BETS) ${ }_{2} \mathrm{FeCl}_{4}$ (Figure 4). The absolute values of the observed Weiss temperature $\theta$ are small, and the observed Curie constants $C$ are nearly equal to spin-only value of $\mathrm{Fe}$ (III) $\left(S={ }^{5} / 2 ; C=4.38\left[\mathrm{emu} \mathrm{mol}^{-1} \mathrm{~K}\right](g=2)\right)$ for both salts. This means that the $\pi$-d interactions in these salts are not so significant as to make a qualitative difference in the band structure from that of the corresponding $\mathrm{GaCl}_{4}$ salts. In other words, the basic band structures are considered to be the same whether $\mathrm{M}=\mathrm{Ga}$ or $\mathrm{Fe}$ in $\kappa$ - and $\lambda$-(BETS $)_{2} \mathrm{MCl}_{4}$, respectively. Therefore the discussion on the many-body effect is considered to be also valid for $\kappa$ - and $\lambda$-(BETS $)_{2} \mathrm{FeCl}_{4}$. This interpretation is consistent with the observed carrier dynamics ${ }^{16}$ and resistivity behavior. $^{3,4}$

3.2. Carrier Dynamics. Two Temperature Model. First, we will compare the carrier dynamics with the so-called two-temperature model (TTM; Figure 5). ${ }^{23-25}$ The TTM is a generally accepted model to describe the energy relaxation dynamics in metals and is based on the assumption that electrons and lattice can be described by two different time-dependent temperature $T_{\mathrm{e}}$ and $T_{1}$, implying that the two subsystem, respectively, each have thermal distribution. In the framework of TTM, e-e thermalization should be much faster than $e$-ph thermalization. After photoexcitation by a sufficiently short laser pulse, conduction electrons quickly (within tens of femtoseconds) thermalize to an effective temperature $T_{\mathrm{e}}$, which can be much higher than the lattice temperature $T_{\mathrm{b}}$, resulting in Fermi-level smearing (step A). Then electrons thermalize with lattice in a characteristic e-ph thermalization time; typically in the 100 fs to 1 ps range (step B). After tens of femtoseconds, when electrons have thermalized, the changes in the occupied electronic density of states are limited to energies of $k_{\mathrm{B}} T_{\mathrm{e}}$ from the Fermi level. The photoinduced reflectivity changes $\Delta R$ tracks the time evolution of the electronic temperature $T_{\mathrm{e}}$.

Amplitude A. The observed carrier dynamics of all the BETS salts is well reproduced by a single-component model

$$
\Delta R(t)=A \exp \left(-\frac{t}{\tau}\right)
$$

where $A$ and $\tau$ imply amplitude and relaxation time of the carriers, respectively. TTM predicts that the amplitude $A$ of the fast electronic transient in step B should monotonously increase 


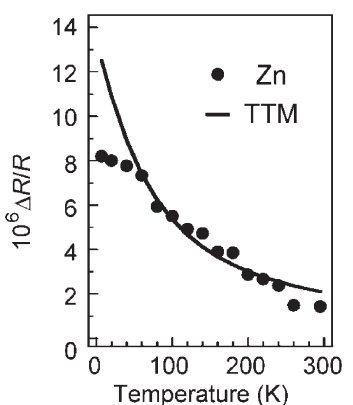

Figure 6. Temperature dependences of amplitude $A$ shown as transient reflectivity change normalized by reflectivity in equilibrium state $\Delta R / R$; expected behavior from standard TTM model, and observed behavior of $\mathrm{Zn}$. Scanned and modified from ref 24 with a copyright permission from APS.
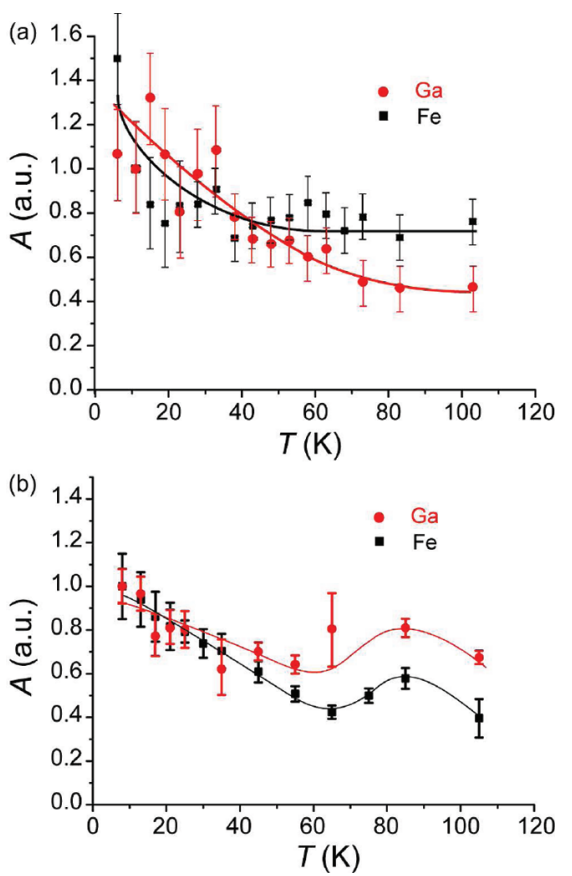

Figure 7. Temperature dependences of amplitude $A$ in transient reflectivity change of (a) $\kappa$ - and (b) $\lambda$-(BETS $)_{2} \mathrm{MCl}_{4}(\mathrm{M}=\mathrm{Ga}, \mathrm{Fe})$.

with decreasing temperature (Figure 6). ${ }^{24}$ This trend qualitatively agrees with the observed behavior of $A$ of both $\kappa$-type BETS salts (Figure 7a). Thus we should assume that the behavior of $\kappa$-type BETS salts can be explained by TTM. In the meantime, a detailed study showed that TTM did not hold for low temperature region $(\leq 50 \mathrm{~K})$ in metals such as $\mathrm{Zn}, \mathrm{Cd}, \mathrm{Au}$, and $\mathrm{Ag}$ (we should tentatively call them "standard metals" below), since $e$-e thermalization time was considered to become comparable or even longer than the $e$-ph thermalization time at low temperature in these metals. ${ }^{24}$ Below $50 \mathrm{~K} A$ in these standard metals exhibited saturation after a monotonic increase with decreasing temperature. The observed behavior of $A$ of both $\lambda$-type BETS salts exhibit only a small temperaturedependence, and we could not definitely tell whether it saturates or not at low temperature (Figure $7 \mathrm{~b}$ ). Thus in the $\lambda$-type BETS salts we should compare the behavior of $\tau$, instead of $A$, with that of TTM.

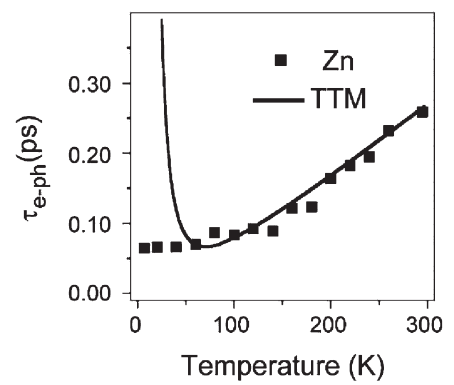

Figure 8. Temperature dependences of electron-phonon thermalization time $\tau_{\text {e-ph }}$, expected behavior from standard TTM model, and observed behavior of $\mathrm{Zn}$. Scanned and modified from ref 24 with a copyright permission from APS.
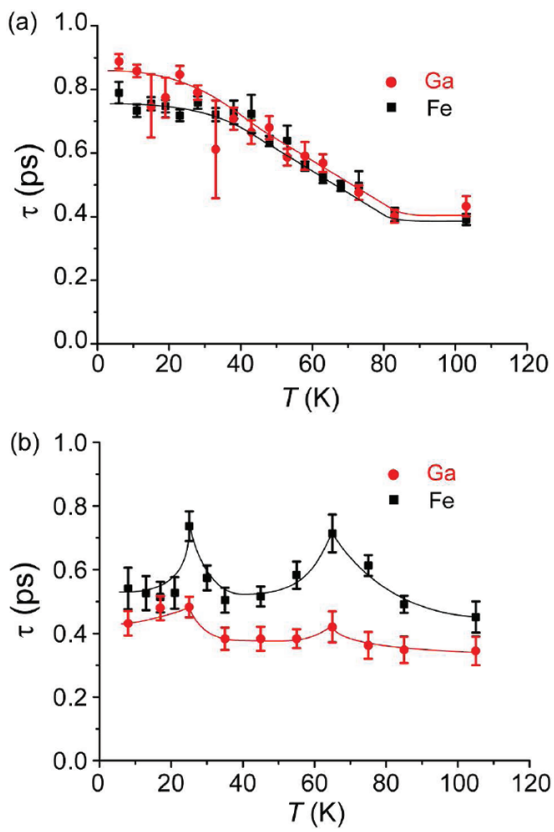

Figure 9. Temperature dependences of relaxation time $\tau$ in transient reflectivity change of (a) $\kappa$ - and (b) $\lambda$-(BETS $)_{2} \mathrm{MCl}_{4}(\mathrm{M}=\mathrm{Ga}, \mathrm{Fe})$.

Relaxation Time of Carriers $\tau$. By assumption of TTM, the observed relaxation is considered to correspond to energy relaxation by $e$-ph thermalization, because the preceding relaxation process by $e$ - $e$ thermalization $(\sim$ tens of femtoseconds) should be too fast to observe with the time resolution of our experiments $(\sim 200 \mathrm{fs})$. In the following discussion, we will compare the relaxation time $\tau$ obtained in our previous work with the $e$-ph thermalization time $\tau_{\mathrm{e}-\mathrm{ph}}$ in TTM.

TTM predicts that $\tau_{\text {e-ph }}$ should gradually decrease with decreasing temperature and should take a minimum around $50 \mathrm{~K}$, below which temperature $\tau_{\mathrm{e}-\mathrm{ph}}$ should increase rapidly with decreasing temperature (Figure 8). ${ }^{24}$ Such behavior is similar to those of both $\kappa$-type salts, though the increase in low temperature region is not as rapid as TTM predicts (Figure 9a). Therefore both $\tau$ and $A$ can be consistently understood by TTM in a qualitative way in $\kappa$-(BETS $)_{2} \mathrm{MCl}_{4}(\mathrm{M}=\mathrm{Ga}, \mathrm{Fe})$. In contrast, $\tau$ of $\lambda$-(BETS $)_{2} \mathrm{MCl}_{4}(\mathrm{M}=\mathrm{Ga}, \mathrm{Fe})$ exhibited nearly temperatureindependent behavior at ca. 7-110 K (Figure 9b), which was clearly different from what is expected from TTM but was rather similar to that of standard metals. At low temperature $(\leq 50 \mathrm{~K}) \tau$ 

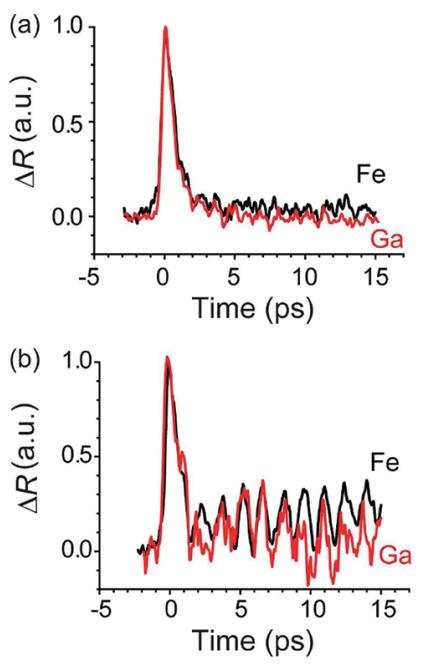

Figure 10. Transient reflectivity change $\Delta R$ for (a) $\kappa$ - and (b) $\lambda$-(BETS $)_{2} \mathrm{MCl}_{4}(\mathrm{M}=\mathrm{Ga}, \mathrm{Fe})$. Coherent phonon response is observed only in (b) as an oscillatory structure.

in standard metals becomes nearly constant, which can be quantitatively explained by nonthermal electron model. ${ }^{23}$ The observed difference in temperature dependence of $\tau$ between $\lambda$ - and $\kappa$-(BETS $)_{2} \mathrm{MCl}_{4}(\mathrm{M}=\mathrm{Ga}, \mathrm{Fe})$ is at least partly due to the difference in strength of $e$-ph interaction between $\lambda$ - and $\kappa$-type BETS salts. In $\kappa$-(BETS $)_{2} \mathrm{MCl}_{4}(\mathrm{M}=\mathrm{Ga}, \mathrm{Fe})$, the $e$-ph interaction is so weak that one can observe an increase in $\tau$ at low temperature in accordance with TTM, while such behavior was not observed in $\lambda$-(BETS $)_{2} \mathrm{MCl}_{4}(\mathrm{M}=\mathrm{Ga}, \mathrm{Fe})$. We should note here that coherent phonons, which exemplify the $e$-ph interaction, were observed at $\leq 50 \mathrm{~K}$ in the $\lambda$-type BETS salts but not in the $\kappa$-type BETS salts (Figure 10). ${ }^{16}$ This can be explained as follows; in the $\lambda$-type salts, the $e$-ph interaction is so strong ${ }^{26}$ that energy relaxation by e-ph thermalization is much faster than TTM assumes. This is consistent with the observed magnetic susceptibility $\chi$ (Pauli) discussed above and also consistent with much higher temperatures of their metal-to-other phase transitions $\left(T_{\mathrm{C}}, T_{\mathrm{N}}\right)$ than those of the $\kappa$-type salts, since the $e$-ph interaction favors such transitions.

\section{CONCLUSION}

The carrier dynamics revealed an important difference in $\kappa$-(BETS $)_{2} \mathrm{MCl}_{4}(\mathrm{M}=\mathrm{Ga}, \mathrm{Fe})$ and $\lambda$-(BETS $)_{2} \mathrm{MCl}_{4}(\mathrm{M}=$ $\mathrm{Ga}, \mathrm{Fe})$; the latter has a strong e-ph interaction peculiar to the crystal structure (molecular arrangement of BETS and $\mathrm{MCl}_{4}$ ). Such classification of carrier dynamics is consistent with clear differences in $T_{\mathrm{C}}\left(T_{\mathrm{N}}\right)$ of them; $T_{\mathrm{C}}\left(T_{\mathrm{N}}\right) \approx 10^{-1} \mathrm{~K}$ for the $\kappa$ - and $T_{\mathrm{C}}\left(T_{\mathrm{N}}\right) \approx 10^{0}-10^{1} \mathrm{~K}$ for the $\lambda$-BETS salts.

\section{ASSOCIATED CONTENT}

S Supporting Information. Details of the tight-binding band calculation, results of single crystal structural analyses, and a summary of magnetic susceptibilities. This material is available free of charge via the Internet at http://pubs.acs.org.

\section{AUTHOR INFORMATION}

\section{Corresponding Author}

*E-mail: tnaito@ehime-u.ac.jp.

\section{Present Addresses}

${ }^{\S}$ Department of Chemistry and Biology, Graduate School of Science and Engineering, Ehime University, Matsuyama, Ehime, 790-8577 Japan.

\section{ACKNOWLEDGMENT}

T.N. thanks Professors T. Nakamura, T. Akutagawa, S. Noro, K. Kubo, Y. Hinatsu, M. Wakeshima, and Y. Doi at Hokkaido University and K. Konishi at Ehime University for their help in SQUID measurements. This work was partly supported by Itoh Science Foundation, The Japan Securities Scholarship Foundation, and Grant-in-Aid for Scientific Research (C) from Japan Society for the Promotion of Science (KAKENHI-23540432).

\section{REFERENCES}

(1) Mori, H. J. Phys. Soc. Jpn. 2006, 75, 051003-051017.

(2) Kato, R.; Kobayashi, H. Synth. Met. 1991, 42, 2093-2096.

(3) Naito, T. Ph.D. Dissertation, The University of Tokyo, Tokyo, Japan, 1995.

(4) Kobayashi, H.; Tomita, H.; Naito, T.; Kobayashi, A.; Sakai, F.; Watanabe, T.; Cassoux, P. J. Am. Chem. Soc. 1996, 118, 368-377.

(5) Hotta, C.; Fukuyama, H. J. Phys. Soc. Jpn. 2000, 69, 2577-2596.

(6) Mori, T.; Katsuhara, M. J. Phys. Soc. Jpn. 2002, 71, 826-844.

(7) Hotta, C. J. Phys. Soc. Jpn. 2003, 72, 840-853.

(8) Kobayashi, H.; Kobayashi, A.; Cassoux, P. Chem. Soc. Rev. 2000, 29, 325-333.

(9) Kobayashi, H.; Cui, H.; Kobayashi, A. Chem. Rev. 2004, 104, 5265-5288.

(10) Uji, S.; Brooks, J. S. J. Phys. Soc. Jpn. 2006, 75, 051014-051023.

(11) Iimori, T.; Naito, T.; Ohta, N. J. Am. Chem. Soc. 2007, 129, 3486-3487.

(12) Iimori, T.; Naito, T.; Ohta, N. J. Phys. Chem. C 2009, 113, 4654-4661.

(13) Iimori, T.; Ohta, N.; Naito, T. Appl. Phys. Lett. 2007, 90, $262103-262105$.

(14) Iimori, T.; Naito, T.; Ohta, N. J. Phys. Chem. C 2010, 114, 9070-9075.

(15) Iimori, T.; Sabeth, F.; Naito, T.; Ohta, N. J. Phys. Chem. C 2011, $115,23998-24003$.

(16) Naito, T.; Matsuo, S.; Inabe, T.; Toda, Y. J. Phys.: Condens. Matter 2010, 22, 185701-185707.

(17) Naito, T.; Fukusaku, M.; Matsuo, S.; Tajima, K.; Inabe, T.; Toda, Y. Physica B 2010, 405, S269-S272.

(18) Otsuka, T.; Kobayashi, A.; Miyamoto, Y.; Kiuchi, J.; Wada, N.; Ojima, E.; Fujiwara, H.; Kobayashi, H. Chem. Lett. 2000, 29, 732-733.

(19) Kobayashi, A.; Udagawa, T.; Tomita, H.; Naito, T.; Kobayashi, H. Chem. Lett. 1993, 22, 2179-2182.

(20) Cui, H.; Brooks, J.; Graf, D.; Okano, Y.; Sun, H.; Kobayashi, H. Inorg. Chem. 2009, 48, 4268-4270.

(21) Freeware downloaded from http://www.primec.com/, PrimeColor Software, Inc.

(22) Kagaku Binran, Kisohen II, 5th ed.; Maruzen: Tokyo, 2004; Chapter 13, pp 631-632.

(23) Groeneveld, R. H. M.; Sprik, R.; Lagendijk, A. Phys. Rev. B 1995, $51,11433-11445$. The quantitative explanation using nonthermal electron model is beyond the scope of this paper and not necessary for the discussion.

(24) Hase, M.; Ishioka, K.; Demsar, J.; Ushida, K.; Kitajima, M. Phys. Rev. B 2005, 71, 184301-184309.

(25) Averitt, D. R.; Taylor, A. J. J. Phys.: Condens. Matter 2002, 14, R1357-R1390.

(26) This can be mainly because the anions are located much closer to the donor (BETS) molecules than they are in the $\kappa$-type salts, which is clear from X-ray structural analyses (see Tables S12-S15). 